\title{
Influencia de las Competencias Emocionales y Financieras en la Formación Universitaria
}

\author{
Ascensión Palomares-Ruiz ${ }^{(1)}$ e Isabel Serrano-Marugán ${ }^{(2)}$ \\ (1) Universidad de Castilla-La Mancha (UCLM), España. Departamento de Pedagogía, Facultad de \\ Educación, Plaza de la Universidad, 3. Albacete, España (e-mail: Ascension.Palomares@uclm.es). \\ (2) Universidad Nacional de Educación a Distancia (UNED), Facultad de Psicología, España \\ (e-mail: Isaserrano@madrid.uned.es)
}

Recibido Ene. 25, 2016; Aceptado Mar. 16, 2016; Versión final Mar. 25, 2016, Publicado Oct. 2016

\begin{abstract}
Resumen
El objetivo del trabajo que se presenta es analizar la importancia de la competencia financiera vinculada con las competencias emocionales. El trabajo posee un enfoque socio-crítico, basado en una metodología fundamentalmente descriptiva, en el contexto de una propuesta de innovación y mejora de la docencia universitaria en la Universidad de Castilla-La Mancha (España). Los recortes que se están produciendo en los sistemas de ayudas públicas, los cambios en los perfiles demográficos y las transformaciones del mundo económico, favorecen una mayor preocupación por el nivel de competencia financiera, no sólo para mejorar la calidad de la educación, sino también como fuente generadora y corresponsable de problemas personales y sociales. Los resultados revelan la importancia de dichas competencias en la formación del profesorado universitario para preparar ciudadanos comprometidos con un mundo más democrático y solidario. Basándose en los resultados del estudio se concluye que un buen docente es aquel que sabe captar las emociones de sus alumnos y es capaz de conducirlas hacia el éxito en sus vidas.
\end{abstract} educativa

\section{Influence of Emotional Competence and Financial Competence in University Education}

\begin{abstract}
The aim of the present work is to analyze the importance of financial competence linked to emotional competencies. The work has a socio-critical approach, based on a fundamentally descriptive methodology in the context of a proposal for innovation and improvement of university teaching at the University of CastillaLa Mancha (Spain). The financial reduction that is occurring in the public aid systems, the changes in demographic profiles and the transformations in the economic world, favor the greater concern about the level of financial competence, not only to improve the quality of education, but also as a generating and coresponsible source of personal and social problems. The results reveal the importance of these competences in the training of university teachers to prepare citizens committed to a more democratic and solidary world. Based on the results of the study it is concluded that a good teacher is that who knows how to capture the emotions of his/her students and is capable of conducting them to have success in their life.
\end{abstract}

Keywords: university education; emotional competence; quality of education; educational innovation 


\section{INTRODUCCIÓN}

En las últimas décadas, los desafíos de la economía mundial demandan a todos los países la adquisición de herramientas creativas e innovadoras para adaptarse y prosperar. Consecuentemente, la Unión Europea (UE) en la Estrategia Educación y Formación 2020, como se refleja en el Informe Español (Ministerio de Educación, Cultura y Deporte, 2013), apuesta por el crecimiento desde una perspectiva ecológica, con cohesión social y territorial, con una económica que haga un uso más eficaz de los recursos, más verde y competitiva. Para ello, es preciso incrementar la innovación y la creatividad, incluyendo el espíritu emprendedor y el desarrollo integral de las personas, desde una perspectiva internacional, con equipos de trabajo eficaces, en continuo proceso de formación, aumentando el valor de las competencias emocionales, porque el incremento de las presiones competitivas otorga un papel significativo a las personas motivadas, creativas, con iniciativa, optimistas y con deseos de superar las dificultades y conflictos, y capacidad para trabajar en equipo.

En el contexto educativo, se ha producido un gran interés por conceptualizar el término competencia; sin embargo, en un principio, la mayoría de los estudios se han centrado en las competencias profesionales. La preeminencia de la formación en competencias ha sido destacada por diferentes instituciones internacionales como la Unión Europea (UE), la Organización para la Cooperación Económica y el Desarrollo (OECD) y la Organización Internacional del Trabajo (OIT), constándose un claro interés en lo que se ha denominado el enfoque de competencias. Con todo, el concepto competencia, en el ámbito educativo, ha ido adquiriendo una conceptualización más integral, por lo que supone una combinación multifuncional y transferible de habilidades prácticas, conocimientos, motivación para aprender, valores éticos, actitudes, aptitudes, emociones y otros componentes sociales y de comportamiento que se organizan y movilizan conjuntamente para lograr una acción eficaz que todas las personas necesitan para su realización y desarrollo personal, inclusión y empleo. Es, por tanto, la capacidad de responder a demandas complejas y llevar a cabo tareas diversas de forma adecuada para comprender e intervenir en todos los campos 0 ámbitos vitales en los que se desenvuelve a lo largo de la vida, de forma equilibrada, activa, creativa y crítica (Palomares, 2014)

Consecuentemente, se están realizando investigaciones, en diversos campos del conocimiento, que confirman la importancia e influencia de las emociones para un adecuado desarrollo y adaptación social de las personas (Gardner, 1985, Goleman, 1995; Damasio, 2000; Cassasus, 2006; Bernal y Cárdenas, 2009; Nelis et al., 2011; González et al. 2011, Palomares, 2014, etc.). Razonadamente, una de las líneas de investigación que mayor interés ha suscitado ha sido el papel que tiene la Inteligencia Emocional (IE) en el contexto educativo y, más concretamente, en su influencia en el éxito académico y la adaptación social del alumnado (Ambrona, López-Pérez y Márquez-González, 2012; Suberviola-Ovejas, 2012)

Goleman (1995) manifiesta que la inteligencia emocional es una forma de interactuar con el mundo, valora los sentimientos y engloba habilidades como el control de los impulsos, la autoconciencia, la motivación, el entusiasmo, la perseverancia, la empatía, la agilidad mental, etc. que configuraran rasgos de carácter, como la autodisciplina, la compasión o el altruismo, indispensables para una buena y creativa adaptación social. Asimismo, este autor, considera la competencia emocional como la capacidad para reconocer nuestros propios sentimientos y los ajenos, con capacidad para manejar de manera positiva las emociones, especialmente aquellas que tienen que ver con las relaciones humanas. Es decir, incorpora capacidades y habilidades.

Desde nuestra perspectiva, definimos la inteligencia emocional como la capacidad de una persona para sentir, entender, disfrutar, controlar y modificar estados anímicos en uno mismo y en los demás. Efectivamente, la inteligencia emocional proporciona a las personas la capacidad y habilidad para regular sus fuerzas e impulsos emocionales, dirigiéndolos y equilibrándolos (Palomares, 2014). Razonadamente, valoramos que las competencias emocionales constituyen el conjunto y entramado de habilidades, conocimientos y actitudes que posee una persona para comprender, expresar, regular, potenciar y promover su inteligencia emocional para conseguir ser una persona digna de forma holística y ecológica; y convivir responsable y pacíficamente. Esta conceptualización también introduce un elemento básico a considerar: el contexto. Consecuentemente, el tiempo y el lugar condicionan las competencias emocionales y la forma en que se aplican en el contexto en que se vive. En esta línea, con el fin de analizar las características básicas de una persona emocionalmente competente, planteamos la necesidad de considerar el contexto socio-cultural y las vivencias personales, como pilares clave; así como la automotivación y las potencialidades de cada persona. Por ello, un profesional competente es el que conoce y regula sus propios procesos de conocimiento, tanto desde el punto de vista cognitivo como emocional, y puede hacer uso estratégico de los mismos, ajustándolos a las circunstancias específicas del problema 0 situación a la que se enfrenta. 
Coincidimos con Marina (2005) y Gardner (2011) en la necesidad de fomentar la "dignidad personal" y el "compromiso social", como sentimientos éticamente construidos y dirigidos, pues debemos ser conscientes de nuestra dignidad y madurez, protegerlas y actuar en consecuencia. Sin embargo, como se ha observado, los docentes españoles se incorporan a los centros educativos con una mochila de conocimientos y habilidades generales propias de la actividad cognitivo-racional por su formación eminentemente académica, pese a no poseer un desarrollo adecuado de sus competencias emocionales.

Como es conocido, el Programa para la Evaluación Internacional de Alumnos (PISA) es un estudio trienal que evalúa en qué medida los alumnos de 15 años que se encuentran finalizando la educación obligatoria han conseguido las competencias y habilidades necesarias para una participación plena en la sociedad. Se evalúa no sólo si los alumnos pueden reproducir los conocimientos adquiridos, sino -también- si pueden utilizarlos y aplicarlos a otros contextos y situaciones. Es decir, en cada una de las preguntas subyace una capacidad y consta de una destreza, un contenido y un método. Es, por tanto, un modelo en el que se pretende evaluar un conjunto de competencias necesarias para participar activamente en la sociedad y cuyo aprendizaje se desarrollará a lo largo de la vida. Se ofrece información muy interesante para la política y la práctica educativa, propiciando una reflexión crítica sobre el conocimiento y habilidades que poseen los alumnos de cada país participante, comparados con los de otros países; y favorece el diseño de metas a conseguir partiendo del análisis de otros sistemas educativos y aprendiendo de las políticas, programas y prácticas implementadas con mejores rendimientos.

La encuesta PISA 2012 (OECD, 2014) se centró en matemáticas, con lectura, ciencia y solución de problemas como áreas menores de evaluación, participando unos 510.000 alumnos/as representando a unos 28 millones de jóvenes de 15 años de 65 países. Por primera vez, de forma opcional, se incluyó una evaluación sobre la competencia financiera que evalúa conocimientos relativos a finanzas cotidianas relevantes para el alumnado de 15 años. España ha participado, junto a otros 17 países, en este primer estudio internacional. Coincidiendo con la publicación de la OECD del Informe Internacional PISA Competencia Financiera, el Instituto Nacional de Evaluación Educativa (INEE) y el Centro de Innovación e Investigación Educativa (CNIIE), organizaron, en 2014, el Congreso PISA Finanzas para la vida que tuvo como objetivo analizar los resultados de los alumnos españoles y las conclusiones del informe, con aportaciones de investigadores y ejemplos de buenas prácticas para propiciar la reflexión y generar propuestas de cambio que mejoren la situación actual.

Resulta necesario subrayar que, la inadecuada formación en competencia financiera, se ha convertido en uno de los factores que inciden en la toma de decisiones financieras desacertadas, haciéndose evidente ante la crisis económica y social en la que estamos abocados. Por ello, dicha competencia es reconocida como uno de los elementos básicos del desarrollo y estabilidad económicos, convirtiéndose en una habilidad esencial para la vida. Consecuentemente, la OECD (2005) recomendó que, la educación financiera se inicie lo antes posible, con el objetivo de que todas las personas puedan recibir una educación sobre cuestiones financieras, subrayando la importancia de centrarse en los jóvenes y la eficacia de impartir educación financiera en los centros escolares.

Asimismo, se ha evidenciado que existe una relación entre la competencia financiera y el contexto familiar económico y educativo de las personas. Como indican Lusardi, Mictchell y Curto (2010), las personas con competencia financiera más elevada proceden de entornos con altos niveles educativos y de familias que manejan numerosos productos financieros. Efectivamente, si se pretende avanzar en la igualdad de oportunidades y en la eliminación de discriminaciones socio-económicas, resulta preciso ofrecer una educación financiera a todo el alumnado desde la más temprana edad, formando ciudadanos más cualificados y dotados para enfrentarse a los nuevos retos de la sociedad en la que viven.

En España, los Centros tienen autonomía para impartir los contenidos del Programa de Educación Financiera de la forma que mejor se ajuste a sus características y necesidades. Por ello, pueden ser tratados como un tema transversal, integrados en otras áreas de conocimiento (Matemáticas, Ciencias Sociales, etc.) o en otras asignaturas como Fomento del Emprendimiento. En el curso 2010-11, participaron 32 Centros y 2.700 alumnos/as; en el curso 2012-13, fueron 400 centros y 21.500 alumnos/as; en el curso 2013-14, participaron 452 centros y 43.000 alumnos/as. Desde nuestra perspectiva, valoramos que la competencia financiera, es una competencia con identidad propia que está muy relacionada con otras competencias, como las emocionales. En esta línea, en el contexto internacional, la OECD, consciente del bajo nivel de competencia financiera de la ciudadanía, desde el año 2002, intentó buscar respuestas para mejorar la situación, llevándose a cabo la mayoría de las iniciativas comunitarias, entre 2007 y 2010.

La Comisión Nacional de Mercado de Valores (CNMV) y el Banco de España, de acuerdo con las recomendaciones y principios de la Comisión Europea y de la OECD, firmaron en 2008 un Convenio de colaboración denominado "Plan de Educación Financiera", con el fin de mejorar la cultura financiera de los 
ciudadanos, dotándoles de herramientas y habilidades para poder adoptar decisiones adecuadas sobre el particular. Dentro de este Plan, uno de los retos era introducir la educación financiera en los Centros de enseñanza y, pocos meses después, se firmó el Convenio con el Ministerio de Educación, denominado Programa Piloto de Educación Financiera, con el objetivo de formar al alumnado de Educación Secundaria. Posteriormente, en el curso 2010-11, el Ministerio de Educación seleccionó a 32 Centros de las Comunidades Autónomas y Ceuta y Melilla que aplicaron el programa de la materia en Ciencias Sociales de $3^{\circ}$ de la ESO. En el curso 2012-13, la experiencia se extendió a 413 Centros, participando un total de 21.500 alumnos de $3^{\circ}$ de la ESO, en la correspondiente evaluación se concluye que el Programa ha sido eficaz en la media que ha permitido a los estudiantes adquirir competencias y conocimientos en el área trabajada. Por ello, se renovó el compromiso y se amplió el Plan hasta 2017. Dicho Programa está integrado en el Proyecto de Emprendimiento del Ministerio de Educación, Cultura y Deporte, diseñado y desarrollado desde el CNIIE, con el fin de incrementar la creatividad y la innovación, incluido el espíritu empresarial, en todos los niveles de la educación y la formación, de acuerdo con uno de los objetivos establecidos en el marco Estratégico Europeo de Educación y Formación (ET2020). Dicho Plan "tiene como objetivo general mejorar la cultura financiera de la población, a fin de que los ciudadanos estén en condiciones de afrontar el nuevo contexto financiero con suficiente confianza" (CNMV y Banco de España, 2013: 7)

La Ley Orgánica de Educación (LOE, 2006), modificada por la controvertida LOMCE (2013), introduce en sus currículos la enseñanza financiera, en Educación Primaria y en Educación Secundaria Obligatoria (ESO). En Educación Primaria se incardina en el currículo de Ciencias Sociales y se trabajan conceptos básicos en relación con el valor del dinero, el ahorro, presupuestos personales y consumo responsable. En la Educación Secundaria Obligatoria se trabaja la educación financiera de forma más específica, por lo que se introducen la economía y la iniciación a la actividad emprendedora y empresarial, incluyendo también contenidos de corte financiero en otras materias. Así, desde el curso 2010/11, se viene desarrollando el programa "Educación Financiera en las Aulas", con un contenido mínimo de 10 horas de enseñanza de conceptos básicos financieros. Resulta necesario indicar que, la competencia financiera es el conjunto de conocimientos, destrezas y estrategias en expansión que los individuos construyen a lo largo de la vida, propiciando la movilización de capacidades cognitivas y prácticas, así como otros recursos (actitudes, motivación y valores). Por tanto, hace referencia al conocimiento y comprensión de conceptos y riesgos financieros; procesos cognitivos genéricos como acceso a la información, comparación y contraste, la extrapolación y la valoración; aplicados a un contexto financiero (destrezas relacionadas con la competencia matemática y la lingüística). Además, como ya hemos indicado, está relacionada con las competencias emocionales, pues se precisa motivación para buscar información y asesoramiento, confianza para hacerlo y capacidad de gestionar factores emocionales y psicológicos que influyen en la toma de decisiones; capacidad para activar y aplicar el conocimiento y la comprensión en situaciones de la vida real y cotidiana; y evidencia el modo en que los individuos gestionan, comprenden y planifican los asuntos financieros propios y los de su entorno. Es decir, las personas con un alto nivel de competencia financiera están mejor preparadas para tomar decisiones solidarias y positivas y, al tiempo, criticar reflexivamente al mundo socioeconómico en el que viven, propiciando los cambios precisos para generar una sociedad más justa y ecológica, sin ningún tipo de discriminación. Por ello, valoramos que la competencia financiera es una destreza clave para la vida y constituye un elemento fundamental en la estabilidad y el desarrollo social, económico y financiero, no sólo personal, sino también del contexto próximo y de la sociedad en la que se vive.

Diversos autores (Schuchardt et al., 2009), resaltan la importancia de la competencia financiera; sin embargo, no existen criterios fiables entre los investigadores a la hora de identificar lo que significa estar "financieramente formado". Razonadamente, hemos considerado muy importante conocer si los futuros docentes están concienciados de la importancia de la competencia financiera y si evidencian que han recibido la formación adecuada.

En los últimos cursos, las Universidades españolas han intentado -con grandes dificultades, entre ellas las económicas- afrontar la definición e implantación de los nuevos títulos de Grado establecidos según las directrices del Espacio Europeo de Educación Superior. En el curso 2010/11, se establece la generalización de la oferta universitaria según los nuevos criterios establecidos en el RD 1393/2007 (Palomares, 2007). En estos momentos en los que se habla de un nuevo cambio en la legislación y normativa al respecto, consideramos oportuno reflexionar críticamente sobre la selección y formación del profesorado de los niveles no universitarios y su posible influencia en los malos resultados que obtiene el alumnado en los Informes PISA. La principal novedad respecto a los planes de estudio anteriores, según las directrices europeas, es que el perfil del titulado debe definirse mediante competencias que debe manifestar al finalizar los estudios. Este paradigma ha supuesto cambios importantes en el proceso de aprendizaje del alumnado y en el papel del profesorado que, necesariamente, debe reorientar su propia formación y actuación. 
Consecuentes con la situación social, económica y educativa en la que nos encontramos, la investigación que se presenta parte de la necesidad de fomentar las competencias emocionales de los docentes para que puedan afrontar y valorar sus propias emociones a nivel personal y profesional con inteligencia emocional y, además, aprender a escuchar, percibir e interpretar las expresiones emocionales del alumnado, ensamblando los sentimientos de la mano de la razón, para tejer una moral sólida como personas y conseguir una auténtica educación inclusiva. Además, en la sociedad en que vivimos, caracterizada por la progresiva complejidad de los mercados y de las relaciones financieras, resulta preciso relacionar las competencias emocionales con la competencia financiera, sin abandonar -en ningún caso- el adecuado desarrollo de todas las competencias para conseguir la formación integral de las personas. Por ello, se plantea el siguiente problema: ¿Los docentes son competentes para formar a sus alumnos para enfrentarse a los retos del futuro, sean capaces de analizar, razonar y comunicar sus ideas de forma eficaz, y desarrollarse como ciudadanos responsables y productivos en la sociedad? Razonablemente, en este trabajo, se plantean como objetivos específicos: a) reflexionar sobre la importancia de las competencias emocionales y la competencia financiera en España; b) analizar la importancia de la competencia financiera en la formación del profesorado en Castilla-La Mancha (España); y c) realizar propuestas para impulsar el desarrollo de las competencias emocionales y de la competencia financiera en el profesorado.

\section{MÉTODO}

El estudio realizado posee un enfoque socio-crítico (Popkewitz, 1988), pues se pretende sacar a la luz supuestos y premisas implícitos en la vida social y educativa para transformar y mejorar la calidad de la formación del profesorado. La actuación práctica de nuestra investigación empírica se basa en un enfoque mixto o "eclético", en la que caben, tanto la investigación cuantitativa, como la de tipo cualitativo. Coincidimos con Erickan y Roth (2006), en el sentido que ambas modalidades deben constituir fases de un proceso unitario, que trata de obtener la información más adecuada para el propósito que nos hemos planteado. Por tanto, este enfoque integrador nos posibilita combinar la predicción con la interpretación e, incluso, con la reflexión crítica liberadora. Los objetivos planteados han determinado la utilización de las modalidades de investigación manejadas. En un principio, debido a que este estudio se inicia en un proyecto de innovación y mejora de la calidad de la docencia universitaria, se han realizado conversaciones o coloquios, en la modalidad de grupos de discusión sobre los objetivos, procedimientos, técnicas e instrumentos posibles a utilizar, empleando una metodología de tipo cualitativo. Es, por tanto, una investigación fundamentalmente descriptiva o interpretativa, pues tratamos de conocer la situación actual en relación con la formación del profesorado en competencias básicas, centrándonos en las competencias emocionales y la competencia financiera, en el momento en que se realiza el estudio, pues nuestro propósito es realizar propuestas de mejora en la formación del profesorado.

\section{INSTRUMENTOS}

En primer lugar, como base del trabajo, se han analizado las diversas publicaciones y estudios realizados sobre el tema. Además, se han considerado adecuadamente los trabajos de innovación y mejora de la docencia ya iniciados en el curso 2012-13, para valorar las actividades realizadas sobre la competencia emocional (Palomares y Alarcón, 2015). Asimismo, se diseñó un cuestionario específico para esta investigación que, con las mejoras propuestas por los expertos, se volvió a pasar los cursos 2013-14 y 2014-15, tras la realización de la actividad diseñada al efecto (elección y justificación de una "Noticia"). Además, se estimó necesario completar dicho instrumento que permitiera recoger la opinión de los futuros docentes en competencias, centrándonos en las emocionales y en la financiera.

La finalidad principal del cuestionario es una recogida de datos válida, fiable y rigurosa para disponer de información sobre la formación del profesorado en competencias, especialmente en las competencias emocionales y en la competencia financiera. La validación del cuestionario fue realizada por 6 expertos seleccionados de manera intencional, procurando que existiera paridad de sexos. Los criterios básicos de validación fueron: relevancia, pertinencia y univocidad. Conforme a lo especificado por Guba (1989), se puede decir que la investigación reúne los cuatro criterios de rigor: valor de la verdad (credibilidad), aplicabilidad (transferibilidad), consistencia (dependencia) y neutralidad (confiabilidad). En el cuestionario se especifican las competencias básicas relacionadas con la competencia financiera y se solicita una valoración sobre la evidencia en que dichas competencias se han trabajado en su formación como docentes, expresando su grado de acuerdo en cada una de las cuestiones, según una escala tipo Likert (Totalmente de acuerdo: 5, Muy de acuerdo: 4; De acuerdo: 3; Desacuerdo: 2; Totalmente desacuerdo: 1; No sabe/No responde: 0 )

Una vez aplicado el cuestionario, la recogida de datos y el tratamiento de los mismos se ha realizado con el programa estadístico SPSS (Statistical Package for the Social Sciences), en su versión 20.0. Este programa ha permitido calcular algunos índices de consistencia científica, tales como el coeficiente de fiabilidad del 
instrumento utilizado. Asimismo, los objetivos planteados en la investigación han requerido el empleo de un enfoque eminentemente cualitativo, toda vez que los datos obtenidos en el cuestionario han sido sometidos a un proceso de reflexión para buscar explicación a los mismos y elaborar propuestas de cambio. Por ello, la parte cualitativa se ha realizado después, con la aplicación de una entrevista semiestructurada concordante con los objetivos de la investigación que nos posibilita enriquecer y profundizar en las respuestas dadas en el citado cuestionario. Se ha formalizado a 16 alumnas y 4 alumnos que han finalizado la Diplomatura de Magisterio y, posteriormente, han cursado el Grado, finalizando en el curso 2014-15 su Trabajo de Fin de Grado. Por ello, las preguntas formuladas, se han centrado en analizar la importancia y la evidencia, a diferencia del cuestionario que sólo se les solicitaba la valoración de la evidencia.

\section{PROCEDIMIENTO}

La investigación efectuada procura ahondar en las posibles causas del fracaso escolar en España y la influencia de la formación del profesorado en competencias emocionales y financiera y, por tanto, puede ser de gran utilidad para introducir cambios significativos en la selección y formación del profesorado aportando justificaciones científicamente fundadas a la política educativa actual, partiendo de la base que, reflexionar sobre el perfil de un docente universitario, supone establecer claramente las características que lo definen e identificar las competencias que debe ostentar con proyección de futuro.

La novedad del estudio radica en el propio propósito que persigue. El trabajo intenta, en la línea también planteada por Pérez y Vila (2013), provocar la inquietud por introducir cambios significativos en la selección y formación inicial y permanente del profesorado, desde una política educativa innovadora y más conexionada con la realidad social y económica en que vivimos.

El contexto de la investigación es la Universidad de Castilla-La Mancha (Facultad de Educación de Albacete) y se ha desarrollado durante los cursos 2012-13, 2013-14 y 2014-15, en el Grado de Maestro, en la Mención de Necesidades Educativas Específicas y Atención a la Diversidad. Ha participado la totalidad del alumnado que configuran los dos grupos (Educación Infantil y Educación Primaria), siendo el 90\% mujeres y un $10 \%$ hombres. De acuerdo con la opción metodología elegida, se han empleado las siguientes técnicas de investigación: análisis de documentos, práctica de una actividad diseñada al efecto: elección y justificación de una "Noticia”, aplicación del cuestionario y realización de entrevistas semiestructuradas.

\section{RESULTADOS Y ANALISIS}

En base a los objetivos planteados, se han analizado trabajos realizados con anterioridad relacionados con la formación del profesorado y examinado la normativa y legislación vigente, así como diversos estudios publicados sobre la formación en competencias. En primer lugar, cabe destacar un dato preocupante, que debe ser centro de atención y respuesta por parte de los responsables políticos: en España la equidad ha empeorado, a pesar de que, las diferencias en rendimiento entre los Centros favorecidos y los menos beneficiados, han sido de 26 puntos frente a los 71 de media de la OECD. En efecto, las diferencias de rendimiento entre los alumnos que proceden de familias más acomodadas y los de menos favorecidas, de un mismo centro educativo, son bastante significativas, aumentando entre 2003 y 2012 , de 18 a 27 puntos, cuando la media en la OECD, en 2012, es de 19 puntos. En 2012, el alumnado con un nivel socioeconómico favorecido supera a los alumnos menos favorecidos en 34 puntos en matemáticas, aumentando en 6 puntos la diferencia establecida en 2003. Además, los alumnos repetidores obtuvieron 102 puntos menos que los no repetidores, lo que supone un aumento de 10 puntos respecto a lo observado en 2003. El rendimiento medio educativo, en España, está por debajo de la media y, lo más preocupante, es que no mejora. Si nos centramos en matemáticas, se sitúa entre los puestos 31 y 36, de los 65 países y economías que participaron en la evaluación PISA 2012, con una puntuación media de 484 puntos, bajando un punto respecto a la obtenida en 2003 (485). En lectura también se alcanzan resultados inferiores a media, situándose ente los países 27 y 35, siendo inamovibles desde el año 2000. Únicamente, en ciencias se ha mejorado ligeramente pasando de 488 puntos, en 2006, a 496, en 2012, situándose entre los puestos 26 y 33.

En resumen, se puede indicar que, si los alumnos de 15 años del 2003 hubieran disfrutado de las mismas características sociales y demográficas que los alumnos de 2012, posiblemente hubieran obtenidos mejores resultados. Este dato debe provocar una reflexión sobre lo que está ocurriendo en el sistema educativo español dado que, a pesar de la mejora del estatus-socioeducativo del alumnado, existe una tendencia negativa en el rendimiento de los alumnos en matemáticas. Es más, si nos centramos en los alumnos que obtienen un rendimiento excelente en matemáticas (8\%), por lo que pueden desarrollar y trabajar modelos para situaciones complejas y trabajar de una forma estratégica utilizando habilidades de pensamiento y razonamiento amplias y bien desarrolladas, nos encontramos con una proporción cinco puntos por debajo de la media de la OECD (13\%), situándose prácticamente inamovible desde 2003 (7.9\%). Además, el 24\% 
del alumnado español que está rezagado en matemáticas, no ha variado desde 2003 (23\%). Otro dato que no ha cambiado es que, en 2012, los chicos obtienen una media de 16 puntos más que las chicas, aumentando la diferencia establecida en matemáticas en 2003, en 7 puntos. Resulta, por tanto, uno de los mayores incrementos observados desde 2003 a 2012, en la diferencia entre chicos y chicas, en todos los países. Por el contrario, las chicas obtuvieron una media de 29 puntos más que los chicos en lectura, manteniéndose estable desde el año 2000, que era de 24 puntos. En ciencias, en el año 2006, los chicos y las chicas alcanzaron unos resultados similares; sin embargo, en 2012, los chicos las superan en 7 puntos. Resulta significativo subrayar que, en España, sólo 19\% del alumnado indica que disfruta leyendo sobre matemáticas, mientras que la media del conjunto de la OECD se sitúa en el $31 \%$. Además, las chicas poseen unos niveles más bajos de motivación intrínseca y, sólo el 14\% disfruta leyendo sobre matemáticas, frente al $36 \%$ de media de la OECD.

Al igual que, en las otras áreas analizadas, el alumnado español obtiene unos rendimientos bajos, pues uno de cada seis alumnos españoles no alcanza el nivel básico de rendimiento en competencia financiera ( $15.3 \%$ de media en los países participantes de la OECD y el 16.5\% en España). Sólo el 3.8\% del alumnado de nuestro país se sitúa en el nivel más alto, con un porcentaje muy inferior al de la media de la OECD (9.7\%). A pesar de que el rendimiento medio de España no es significativamente diferente al de Francia, Croacia, Israel, la Federación Rusa, Eslovenia y los Estados Unidos, se sitúa por debajo de la media de los países participantes entre los puestos 10 y 15, con 484 puntos (Tabla 1)

Tabla 1: Rendimiento medio en competencia financiera (basada en informes de la OECD)

\begin{tabular}{|l|c|c|}
\hline País & Puntuación media & Rango \\
\hline Shanghái-China & 603 & $1-1$ \\
\hline Bélgica (Flandes) & 541 & $2-2$ \\
\hline Estonia & 529 & $3-4$ \\
\hline Australia & 526 & $3-5$ \\
\hline Nueva Zelanda & 520 & $4-6$ \\
\hline República Checa & 513 & $5-7$ \\
\hline Polonia & 510 & $6-7$ \\
\hline Letonia & 501 & $6-7$ \\
\hline Estados Unidos & 492 & $8-12$ \\
\hline Federación Rusa & 486 & $9-14$ \\
\hline Francia & 486 & $9-14$ \\
\hline Eslovenia & 485 & $9-14$ \\
\hline España & 484 & $10-15$ \\
\hline Croacia & 480 & $11-16$ \\
\hline Israel & 476 & $11-17$ \\
\hline República Eslovaca & 470 & $15-17$ \\
\hline Italia & 466 & $16-17$ \\
\hline Colombia & 379 & $18-18$ \\
\hline Media Oecd-13 & 500 & \\
\hline
\end{tabular}

Si comparamos los resultados obtenidos en matemáticas y lectura con los de competencia financiera, sorprende que, los alumnos con buen rendimiento en matemáticas y lectura, no se desenvuelvan de la misma forma en aspectos financieros; mientras que, los alumnos con bajo rendimiento en matemáticas o lectura, obtienen resultados superiores a lo esperado. Resulta importante destacar que, en España, el origen socio-económico afecta más al rendimiento en la competencia financiera que los obtenidos en otras áreas. Estos resultados (15\%) son similares al de la media de la OECD (14\%). Evidentemente, el nivel educativo y la profesión de los padres influyen en los resultados. Así, los alumnos que proceden de familias donde el padre o/y la madre tienen estudios superiores consiguen 39 puntos más, diferencia que aumenta si el padre o/y la madre disfrutan de una profesión cualificada, superando en 52 puntos a los que tienen menor cualificación profesional. Relacionado con lo anteriormente indicado, los alumnos inmigrantes obtienen 52 puntos menos que los nativos (35 puntos si se tiene en cuenta el origen socioeconómico), porcentaje muy superior al de la media de los alumnos inmigrantes de la OECD (37 puntos) 
Resulta interesante subrayar que los resultados en competencia financiera entre los chicos y las chicas son similares, tanto en España como en la media de los países de la OECD. En resumen, los resultados obtenidos en competencia financiera vuelven a ratificar -una vez más- la preocupante situación del sistema educativo español que no se soluciona únicamente con cambios de leyes. Asimismo, hay elementos que reiteran la importancia de una educación de calidad, desde la más temprana edad. Conviene recordar que, entre 2003 y 2012, la diferencia en rendimiento entre quienes han asistido a educación infantil durante más de un año y los que no han asistido se ha incrementado en más de 30 puntos. Otro indicador clave que influye en los resultados es el profesorado, por lo que resulta necesario plantearse: ¿Qué cambios significativos se han introducido desde 2003? ¿Se forma al profesorado para que pueda propiciar el desarrollo de las competencias emocionales y las financieras? Según los resultados del cuestionario aplicado a la muestra de la investigación, en el que se les solicitaba que valoraran de 1-5 la evidencia en la formación de la competencia financiera correlacionada con las competencias emocionales, se comprueba que las valoraciones son muy bajas.

Es preciso indicar que, en la investigación realizada, se ha estudiado la relación de todas las competencias básicas con la competencia financiera; sin embargo, este artículo se centra únicamente en los resultados obtenidos en la relación entre competencias emocionales y la competencia financiera. Razonadamente, se ha estudiado la importancia de la formación del profesorado y su posible influencia en los resultados del alumnado en los Informes PISA. Por ello, se han analizado las conclusiones del Estudio Internacional TALIS (2014), en el que se examinan diversos resultados de la enseñanza y el aprendizaje a través de encuestas a los profesores y directores de los centros educativos. Si nos centramos en España, destaca que, más del $97 \%$ del profesorado, declara sentirse bien preparado para el trabajo docente, porcentaje superior al de la OECD (90\%); porcentaje también similar en lo relativo a los que afirman haber participado en programas de formación específica para ejercer la docencia. Como datos significativos habría que destacar que, 6 de cada 10 profesores, trabajan en Centros en los que faltan profesores para atender al alumnado con necesidades educativas especiales, mientras que en la OECD la proporción es un poco inferior ( 5 de cada 10), el tamaño medio de la clase y la ratio de alumnos/profesor se encuentra en la media del conjunto de los países de la OECD; sin embargo, la tasa de personal de apoyo pedagógico respecto al número de profesores (19) es inferior a la media de la OECD (12); los Centros españoles tienen menos autonomía en la toma de decisiones que los centros de los países participantes de la OECD, encontrándonos entre los países con la media de edad de los directores más jóvenes; con todo, destaca la falta de formación en liderazgo, donde 4 de cada 10 directores señalan que no han recibido esta formación. España se encuentra estancada en niveles por debajo de la media de la OECD y parece que no existe tendencia alguna de mejora, situación que se ha visto influenciada por los diversos cambios legislativos que están provocando una variación continua en la estructura del sistema escolar y una reorganización de los Centros, sin tener tiempo para que puedan aplicarse las diferentes propuestas. Los cambios de Leyes Orgánicas cada cinco o seis años, dificultan la consolidación de un modelo educativo de calidad y, consecuentemente, se precisa apartar la educación del debate partidista. Además, los datos existentes provocan que nos planteemos si la selección y formación inicial del profesorado prepara realmente para las demandas de la sociedad.

Si nos centramos en los resultados de la investigación que hemos realizado en la UCLM (España), una vez descartados los cuestionarios que no cumplimentaban los apartados considerados obligatorios, en el curso 2014-15, se obtuvieron 172 respuestas válidas, de las que 156 eran alumnas y 16 alumnos. Se evidencia, una feminización en los estudios de Grado, en la Mención de Necesidades Educativas Específicas y Atención a la Diversidad, en Educación Infantil y Educación Primaria. A partir de los datos del cuestionario se procedió a realizar un análisis más detallado de las dos competencias en la que se ha centrado este estudio: la competencia emocional y la competencia financiera. La información obtenida, al igual que en los cursos anteriores (2012-13 y 2013-14), revela que, una de las principales causas de los malos resultados del alumnado español, no es la actitud o nivel de satisfacción del profesorado; pues diversos estudios evidencian que los puntos fuertes de su satisfacción laboral residen en las buenas relaciones con los compañeros de trabajo, el sentimiento de que el trabajo es adecuado para ellos y que realizan algo valioso, que es motivador y les permite autonomía en el desarrollo de las actividades. No obstante, como también han concluido las investigaciones realizadas por Anaya y López (2014), consideran como puntos débiles que están lastrando esa satisfacción: el insuficiente reconocimiento económico del rendimiento laboral; la falta de posibilidades de promoción en base del propio rendimiento y habilidades; y el escaso asesoramiento de expertos. Razonadamente, resulta preciso indagar por qué el profesorado español no tiene unos niveles mayores de exigencia en su formación inicial más adaptada a las demandas de la sociedad actual. Si reflexionamos sobre las causas del éxito del sistema educativo de Finlandia, se podría destacar que la clave de la mejora de los últimos treinta años está en la transformación de la selección y formación del profesorado. A diferencia de España, donde los profesores con menos experiencia, tienden a trabajar en mayor proporción en centros educativos de más difícil desempeño y en los niveles más bajos de Educación Primaria (TALIS, 2014), en Finlandia, donde no existe educación infantil reglada como en España de 3 a 6 años, los mejores docentes se sitúan en los primeros años de la enseñanza escolar, 
porque es el momento en que se adquieren los fundamentos de los aprendizajes posteriores. Con todo, diversos estudios (Fernández y Baena, 2015) evidencian que los profesores más satisfechos en España son los que trabajan en la Etapa de Educación Infantil. También resulta interesante subrayar que existe una menor satisfacción global, en 2012-13 respecto a 2003-04.

Un elemento clave del éxito, en Finlandia, está en la selección del profesorado que se realiza antes de comenzar los estudios de magisterio y, "durante el proceso de formación, se estimula al alumno para que integre los conocimientos que debe aprender con el objetivo de desarrollar con éxito su función docente" (Melgarejo, 2014: 121). Lógicamente, si se pretende que sólo los mejores alumnos puedan acceder a las Facultades de Educación, los profesores de las mismas, tienen que ser los mejores de todas las promociones y de las especialidades que enseñan. Por ello, las diferencias de rendimiento entre las diversas escuelas son pequeñas y todo el alumnado aprende bien. Sin embargo, en España, la selección del profesorado se realiza una vez culminados los estudios universitarios, mediante un proceso de oposiciones que permiten el acceso al sistema escolar público, y existen grandes diferencias de rendimiento entre las diferentes escuelas y alumnado. Todo ello, ratifica -una vez más- que resulta preciso un cambio en la selección y formación del profesorado en nuestro país.

\section{DISCUSIÒN FINAL}

Las competencias emocionales dotan al profesorado de mayor confianza en sí mismo y de sus potencialidades, le ayudan a autorregularse, a actuar con creatividad y flexibilidad, a motivarse, tener afán por el logro, reforzando la iniciativa, el compromiso y el optimismo. Además, mejora la empatía y las relaciones con los otros miembros de la comunidad educativa, aporta vocación de servicio y propicia una mayor respuesta a la diversidad, optimizando los procesos de comunicación, a manejar mejor los conflictos y establecer vínculos de cooperación y colaboración, creando un verdadero espíritu de equipo. En resumen, las citadas competencias pueden proporcionar, a los docentes de todos los niveles educativos, las habilidades precisas para conducir al alumnado al éxito, a través del conocimiento de la realidad en que se vive, el autoconocimiento, el autocontrol, la auto-motivación, la empatía y el control de las relaciones sociales y educativas.

Razonadamente, del análisis y discusión de los resultados de la investigación realizada en la UCLM, se puede llegar a una primera conclusión: la insuficiente formación inicial del profesorado de Educación Infantil y de Educación Primaria en competencias emocionales y en competencia financiera. Se constata que las competencias emocionales constituyen un factor clave en el desarrollo de todas las áreas de nuestra vida y determinan nuestras decisiones financieras de manera muy significativa, según han ratificado el $92 \%$ de los encuestados. Además, aprender a conocernos mejor, a saber predecir nuestras reacciones y sentimientos, nos permite evitar situaciones desagradables y favorecer las circunstancias positivas. Al tomar decisiones financieras es trascendental que nuestras emociones estén controladas, aprendiendo a desarrollar nuestra capacidad de análisis y observación de las personas y de las instituciones financieras. Asimismo, no se debe olvidar que no resulta fácil mejorar las competencias emocionales y no existe un modelo único e infalible para lograrlo. Por ello, en los trabajos de innovación y mejora de la calidad de la docencia, se ha procurado implicar al alumnado para que sientan la necesidad de desarrollar y valorar sus competencias emocionales y, al tiempo, realicen propuestas concretas para mejorar su formación, desde una perspectiva integral. De acuerdo con los datos obtenidos, se otorga una gran importancia a las competencias estudiadas; sin embargo, se evidencia una significativa ausencia en su formación, como indican el $83 \%$ de los participantes en la investigación. Otro dato significativo a enfatizar es que todos los encuestados valoran que, en su formación no se otorga la importancia precisa a las competencias emocionales, mostrando su preocupación ante esta situación. Además, los entrevistados añaden que no perciben cambios en el Grado respecto a la Diplomatura, no sólo en la competencia financiera, sino también en las competencias emocionales. Asimismo, también hay que destacar que, la mayoría de los participantes en la experiencia valoran muy positivamente los trabajos realizados sobre la actividad "La Noticia" (que ha servido para el estudio), y valoran que han tomado conciencia de la importancia de las competencias emocionales en su vida personal y en su futuro como docentes, por lo que se insiste en impulsar la formación de dichas competencias, en su proceso formativo de manera transversal, aportando iniciativas para trabajar conjuntamente las competencias emocionales con la financiera.

En similar sentido concluyen las investigaciones realizadas por López-Goñi y Goñi Zabala (2012) subrayando que las competencias emocionales ocupan un lugar menor en el conjunto de competencias docentes, por lo que sería necesaria una mayor atención a las competencias relacionadas con las capacidades emocionales de tipo intrapersonal. En esta línea, se orientan diversas investigaciones subrayando la necesidad de articular actividades que tengan como objetivo la formación de competencias socio-emocionales dentro de la formación integral de profesor novel, basadas en situaciones reales y en estrategias emocionales que utilizan profesores veteranos para enfrentarse a esas situaciones y adaptarse a las mismas, utilizarlas o cambiarlas, a la hora de desarrollar estrategias propias. 
Como hemos resaltado anteriormente, en las valoraciones realizadas por el alumnado, las metodologías activas suponen un impacto positivo en la calidad de las relaciones interpersonales en el aula (Palomares, 2011). Por ello, se propone la elaboración de investigaciones y experiencias que fomenten las competencias emocionales e interpersonales del profesorado en proceso de formación, en la línea también manifestada por autores como Ambrona, López-Pérez y Marquez-González (2012); y Pérez Escoda, Filella, Soldevila y Fondevila (2013). Igualmente, se ha podido comprobar que los participantes en la experiencia (futuros docentes) que valoran más las estrategias activas para enfrentarse a situaciones diversas y novedosas en su proceso formativo, son los que se perciben a sí mismos como poseedores de determinadas habilidades para regular sus emociones y, además, las reconocen y valoran en sus profesores.

Los entrevistados han evidenciado la influencia de las competencias sociales y emocionales en la formación docente como medio para mejorar los procesos de aprendizaje y las relaciones del alumnado, así como su importancia en la prevención del burnout. Una vez más, hay que subrayar que la educación debe atender todas las dimensiones de persona humana y sus relaciones con los demás, y en las exigencias sociales que permiten participar plenamente en una sociedad democrática, por lo que se precisa una atención complementaria a la formación impartida en las diferentes áreas del currículo, como también especifican Palomares y Serrano (2013). Igualmente, hay que destacar que, en este estudio se ha incluido el trabajo colaborativo, aportando conclusiones muy significativas sobre la eficacia de las actividades desarrolladas para promover las competencias emocionales y sociales del alumnado. En este sentido, se coincide con las investigaciones realizadas por Pérez y Vila (2013), al subrayar la importancia del trabajo en grupo para movilizar las capacidades de otras personas. Por ello, en posteriores investigaciones se incidirá en la importancia de trabajar las citadas competencias de forma cooperativa y su influencia en la prevención de la agresividad, el acoso y la violencia escolar.

Asimismo, se evidencia la necesidad de potenciar las emociones con libertad, posibilitando que los sentimientos y pasiones sean las voces que definan y establezcan las pautas de comportamiento y la forma en que las personas se integran en su entorno. Para ello, se propone desarrollar la capacidad para afrontar las dificultades relacionadas con la autoestima y ser competentes para resolver los diversos conflictos y problemas de la vida cotidiana. Coincidimos con Bisquerra (2005) al subrayar que la formación inicial debería dotar al profesorado de un bagaje sólido en competencias emocionales. Por ello, se pretende afrontar dicha tarea de forma holística, por medio de diversas actividades cuyas características esenciales se especifican en este artículo. En las entrevistas realizadas se ha puesto de manifiesto la preocupación e inquietud de los futuros docentes por las consecuencias que puede producir la acentuación de las desigualdades en el sistema educativo español, subrayando la existencia de:

i) Divergencias en la formación del profesorado según el poder adquisitivo, dado que los Máster resultan muy costosos y no todas las personas pueden acceder a ellos.

ii) Diferencias entre los centros en función de su nivel de solicitud, lo que influirá en la demanda de formación y los procesos de selección, en función de sus propios criterios, no siempre acertados y en concordancia con la mejora de la calidad.

iii) Nuevo papel otorgado a los directores de los centros no universitarios (LOMCE, 2013), al primar las estrategias directivas más relacionadas con la gestión empresarial que con la educativa y didáctica, generando inquietud.

iv) Influencia del contexto en que se vive, especialmente por la crisis económica y de valores que invade la sociedad española.

\section{CONCLUSIONES}

De los resultados mostrados, de su análisis y discusión, se puede indicar que la mejora de la calidad de la educación en España requiere que los docentes y los directivos de los Centros desarrollen las competencias emocionales y la competencia financiera, especialmente en la situación social y económica en la que vivimos, inmersos en una crisis económica y de valores sociales.

Se puede concluir también que para ser buen docente se precisa una formación excelente en todos los campos y adaptada a los retos que exige la sociedad.

\section{REFERENCIAS}

Ambrona, T., López-Pérez, B. y Márquez-González, M., Eficacia de un programa de Educación Emocional breve para incrementar la competencia emocional de niños de Educación Primaria, Revista Española de Orientación y Psicopedagogía (REOP), 23(1), 39-49 (2012) 
Anaya, D. y López, R., Satisfacción laboral del profesorado en 2012-13 y comparación con los resultados de 2003-04, Un estudio de ámbito nacional, Revista de Educación, 365, 96-121 (2014)

Bernal, A. y Cárdenas, A.R., Influencia de la competencia emocional docente en la formación de procesos de motivacionales e identitarios en estudiantes de educación secundaria. Una aproximación desde la memoria autobiográfica del alumnado. Revista de Investigación Educativa, 27(1), 203-222 (2009)

Bisquerra, R., La educación emocional en la formación del profesorado, Revista Interuniversitaria de Formación del Profesorado, 19(3), 95-114 (2005)

Cassasus, J., La educación del ser emocional, Santiago de Chile, Índigo (2006)

COMISIÓN NACIONAL DEL MERCADO DE VALORES Y BANCO DE ESPAÑA, Plan de Educación Financiera 2013-2017, Madrid, CNMV y Banco de España (2013)

Damasio, A. R., Sentir lo que sucede. Cuerpo y emoción en la fábrica de la conciencia, Santiago de Chile, Andrés Bello (2000)

Erickan, K. y Roth, W.M., What good is polarizing research into qualitative and quantitative, Educational Researcher, 35(5), 14-23 (2006)

ESTUDIO TALIS 2013, http://www.mecd.gob.es/inee/Ultimos_informes/TALIS-2013.html, Acceso: 25 diciembre (2014)

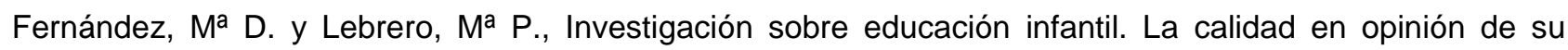
profesorado, Síntesis, Madrid (2015)

Gardner, H., Frames of mind: the theory of multiple intelligences, New York, Basic Books (1985)

Gardner, H., Verdad, belleza y bondad reformuladas, Barcelona, Paidós (2011)

Goleman, D., Inteligencia emocional, Barcelona, Kairós (1995)

González, M. et al., Construcción y Validación preliminar de la Escala de apertura al conocimiento y al Cambio Emocional (EACCE) en Estudiantes Universitarios, Terapia Psicológica, 29(2) (2011)

Guba. E.G., Criterios de credibilidad en la investigación naturalista. En Gimeno, J. y Pérez, A.I. (Eds.), La enseñanza: su teoría y su práctica (148-165), Madrid, Akal (1989)

López-Goñi, I. y Goñi Zabala, J., La competencia emocional en los currículos de formación inicial de los docentes, Revista de Educación, 357, 467-489 (2012)

Lusardi, A., Mitchell, O.S. y Curto, V., Financial literacy among the Young, The Journal of Consumer Affairs, 44(2), 358-380 (2010)

Marina, J.A., Precisiones sobre la educación emocional, Revista interuniversitaria de formación del profesorado, 19(3), 27-43 (2005)

MECD, Ley Orgánica 272006, de 3 de mayo, de Educación, Boletín Oficial del Estado, № 106 de 4 de mayo, 17158-17207 (2006)

MECD, Ley Orgánica 8/2013, de 9 de diciembre, para la Mejora de la Calidad Educativa, Boletín Oficial del Estado, № 295 del 10 de diciembre, 97858-97921 (2013)

MECD, Congreso Finanzas para la Vida. Madrid, 9 y 10 de Julio de 2014, http://www.mecd.gob.es/inee/Informaciones-de-interes/Congreso PISA finanzasparalavida.html, Acceso: 4 de diciembre (2014)

Melgarejo, X., Gracias, Finlandia, Barcelona, Plataforma actual (2014)

Ministerio de Educación, Cultura y Deportes, Objetivos Educativos Europeos y Españoles, Estrategia Educación y Formación 2020, Informe Español, 2013, Madrid, MECD, Secretaría de Estado de Educación, FP y Universidades, (2013), http://www.mecd.gob.es/dctm/inee/...educativos/informeet20202013.pdf, Acceso: 19 de enero (2015) 
Nelis, D. et al., Increasing emotional competence improves psychological and physical well-being, social relationships, and employability, Emotion, 11, 354-366 (2011)

OECD, Recommendation on Principles and Good Practices for Financial Education, Bruselas, OECD Publishing (2005)

OECD/INFE, High-Level Principles on National Strategies for Financial Education, Bruselas, OECD Publishing (2012)

OECD, PISA 2012 Results: What Students Know and Can D. Student performance in Mathematics, Reading and science, OECD Publishing (2014)

Palomares, A., Nuevos retos educativos. El modelo docente en el Espacio Europeo, Cuenca, España, Publicaciones de la UCLM (2007)

Palomares, A., El modelo docente universitario y el uso de nuevas metodologías en la enseñanza, aprendizaje y evaluación, Revista de Educación, 355, 591-604 (2011)

Palomares, A., Innovative methodologies to promote teacher's emotional competences, Journal for Educators, Teachers and Trainers, Vol. 5 (2), 116-129 (2014)

Palomares, A. y Alarcón, M ${ }^{a}$ C., Formación en competencias emocionales y financieras para afrontar nuevos retos de la sociedad, En A. De-Juanas y A. Fernández-García (Coord.), Pedagogia social, universidad y sociedad, 29-36, Madrid, UNED (2015)

Palomares, A. y Serrano, Ma I., La respuesta a la diversidad para una cultura de la paz y la convivencia, En Ma C. Pérez y Ma M. Molero (Coord.), Variables psicológicas y educación para la intervención en el ámbito educativo, 111-115, Almería, Universidad de Almería (2013)

Pérez Escoda, N., Filella, G., Soldevila, A. y Fondevila, A., Evaluación de un programa de educación emocional para Profesorado de Primaria, Educación XX1, 16 (1), 233-254 (2013)

Pérez Vázquez, P.J. y Vila, L. E., La adquisición de competencias para la innovación productiva en la universidad española, Revista de Educación, 361, 492-455 (2013)

Popkewitz, TH. S., Paradigma e ideología en investigación educativa, Las funciones sociales del intelectual. Madrid: Mondadori (1988)

Suberviola-Ovejas, I., Competencia emocional y rendimiento académico en el alumnado universitario, Revista de Comunicación Vivat Academia, Año XIV, № Especial, 1-17 (2012)

Schuchardt, J. et al., Financial Literacy and Education Research Priorities, Journal of Financial Counseling and Planning, 20 (1), 84-95 (2009) 